\title{
Orthopädische Verbandtechnik und Apparatotherapie.
}

\author{
Sammelreferat für 1920. \\ Von \\ Dr. Hans Blencke-Magdeburg, \\ 1. Assistent der orthopädischen Feilanstalt von Prof. Ur. A. Blencke.
}

(Eingegangen ain 19. November 1921.)

In der orthopädischen Verbandtechnik und Apparatotherapie hat das Jahr 1920 keine Veröffentlichungen aufzuweisen, die sich mit dem Gesamtgebiet ausführlich beschüftigen, während kurz gefaßte Übersichten aus der Feder mehrerer Autoren erschienen sind; so widmete Erich Sonntag in seinem Grundriß der gesamten Chirurgie, den er in Form eines Taschenbuchs für Studierende und Ärte herausgab, auch der Verbandlehre ein Kapitel. Rudolf Tinner behandelt in einem kurzen Repetitorium des Seemannschen Verlages, wie sie sich zur Examensworbereitung bestens bewährt haben, gleichfalls das Gebiet der Verbände im Zusammenhang mit Operàtionsübungen, Frakturen und Luxationen.

Der Vervollkommnung des orthopädischen Hilfs- und Krankenpflegepersonals, auf dessen Mitarbeit der Orthopäde vielleicht mehr als mancher andere Zweig der Medizin in entscheidender Weise angewiesen ist, dient ein Lehrbuch Hans Debrunners, in dem er in ausgezeichneter Weise die Verband- und Gipstechnik sowie die Mechanotherapie allgemeinverständlich zur Darstellung bringt. Den gleichen Zwecken dient eine Abhandlung desselben Autors über Tätigkeit und Ausbildung der orthopädischen Hilfsschwester in der Zeitschrift für Krankenpflege, in welcher auch Adam über die Ausbildung von Hilfs. personal in der physikalischen Therapie Stellung nimmt. Jedem Orthopäden, dem an der Weiterbildung seines Hilfspersonals gelegen ist, können diese $A b$. handlungen empfohlen werden.

1. Adam, A., Die Ausbildung von Hilfspersonal in der physikalischen Therapie. Zeitschr. f. Krankenpflege 1920. - 2. Debrunner, Hans, Tätigkeit und Ausbildung der orthopädischen Hilfsschwester. Ebenda. - 3. Dersel be, Lehrbuch für orthopüdische Hilfsarbeiterinnen. Verlag von F. B. W. Vogel in Leipzig 1919, - 4. Sonntag, Erich, Grundriß der gesamten Chirurgie. Ein Taschenbuch fuir Studierende und Ärate. Allgemeine Chirurgie, spezielle Chirurgie, Frakturen und Luxationen, Operationskurs, Verbandlehre. Berlin 1920. Julius Springer. - 5. Tinner, Rudolf, Operationsübungen. Frakturen und Luxationen, Verbände. Berlin 1920. Siegfried Seemann. - 


\section{Verbandtechnik.}

Zur Wundbehandlung, die ja in der operativen Orthopädie eine große Rolle spielt, wird von Scheidt mann Jatrengaze empfohlen, die austrocknend, desodorisierend und granulationsanregend wirkt und den Vorteil bietet, beliebig of sterilisiert werden zu können, da sich das Jatren erst bei $223^{\circ}$ zersetzt. Prop ping will das Verkleben der Gazetampons und Gazedrains mit der Wunde durch Anwendung nicht entfetteter Rohgaze vermeiden, nimmt aber dabei den Nachteil in Kauf, daß die Kapillardrainage der Rohgaze gleirh Null ist. Gretsel hat durch Stanniol und Aluminiumfolien, mit denen er Wundkanäle ausgelegt und die Granulationen bedeckt hat, die Austrocknung der Wunden verhindert und einen schmerzlosen, die Granulationen nicht verletzenden Verbandwechsel erreicht. Die Wirkungsweise der Verbandstoffe in physiologisch-chemischer Hinsicht ist von Belák untersurht worden, wobei er feststellte, daß sie nicht nur rein mechanisch schützend wirken, sondern auch die Eiweißkörperspaltung hemmen und Bakterien und Toxine absorbieren. Als Ersatz des für Behelfsapparate zu teuren Zelluloids empfiehlt Roederer das zur Leimfabrikation verwendete Kaseinpulver, das, mit $1 / 3$ frisch gelöschtem Kalkpulver gemischt, unter Luftabschluß aufzubewahren und beim Gebrauch mit 4 Teilen Wasser und Zusatz von 5-10\% igen Glyzerin in dicker Schicht wie bei der Zelluloidtechnik aufzupinseln ist.

Die bei zu festen Verbünden zu befürchtende Ischämie hat Brücke experimentell studiert; eine reine Fixationskontraktur war am Frosch durch 3-5wöchige Schienenverbände nicht zu erzielen, währen丸 komprimierende Fixationsverbände Gelenkversteifung, Trübung. Starre und schließlich Unerregbarkeit der Muskulatur bewirkten, wofür außer der Hemmung der arteriellen Blutzufuhr die Verhinderung der Lymphzirkulation und die Muskelkompression anzuschuldigen sind.

Die spezielle Verbandtechnik bei den einzelnen orthopädischen Leiden ist durch zahlreiche Arbeiten bereichert worden.

In der Behandlung der Luxatio coxae congenita hat Peltesohn einen federnden, mit aufgenietetem Filz gepolsterten Eisenblechstreifen angegeben, den er nach gelungener Reposition mit Klebelösung und Kambrikbinde fixiert, ein bogenförniges Mittelstück auf dem Kreuzbein, die geraden Schenkel auf den Hinterflächen der Oberschenkel, während die umgebogenen Enden die Knie nach vorn umgreifen. Er will hierdurch die teuer gewordenen Gipsverbände ersparen. Uther die Verbandtechnik der angeborenen Hüftluxation verbreitete sich Fränkel auf dem XV. Orthopädenkongreß, wobei er betonte, daß er der Einrenkung stets die Extension dureh Drahtextension vorausschicke. Die Vorzüge des Schedeschen StoBverbandes, der den Übergang zum verbandlosen Gehen wesentlich erleichtere, rühmt Meyex, der diesen Verband lieber Stampfverband genannt wissen will.

Den angeborenen Klumpfuß behandelt $\mathrm{Frän}$ kel bei Säuglingen mit einem mit einer Spiralfeder armierten Heftpflasterverband, der zum Redressieren dient, wo das Saugapparatredressement nicht anwendbar ist. Ob diese Methode dem manuellen Redressement überlegen ist, sei dahingestellt. Zur Fixierung des redressierten Klumpfußes empfiehlt Veilchenblau einen Bügelgipsverband, der aus einem gut anmodellierten Gipsring am unteren Drittel des Unter- 
schenkels und einem Gipsschuh vom Sprunggelenk bis zu den Zehengrundgelenken besteht. Ein über Schusterspan angelegter Gipsbügel verbindet die Innenseite des oberen mit der Außenseite des unteren Teiles.

Die Osteochondritis deformans coxae juvenilis (Perthes), die jetzt eifrig diskutiert wird, behandelt Brandes zur Entspannung der spastischen Adduktoren mit einem in $25-30^{\circ}$ Abduktion angelegten, ruhigstellenden Gehgipsverband bis zu 12 Wochen Dauer, worauf der Gehbügel abgenommen wird und allmähliche Belastung erfolgt.

Um infolge langen Liegens eines Gipsverbandes etwa zu erwartende Kniegelenksversteifungen zu verhüten, beschreibt $\mathrm{Haubenreißer}$ einen an der Payrschen Klinik gebräuchlichen Kunstgriff, ein Patellarfenster einzuschneiden, welches eine aktive Quadrizeps- und Patellarbewegung ermöglicht, so daß nach Verbandabnahme in 3-4 Tagen volle Beweglichkeit des Knies besteht.

Kleinschmidt hat die Beugekontraktur der Großzehe als eine typische Deformität beschrieben und zu ihrer Beseitigung ein an der Payrschen Klinik ausgearbeitetes Operationsverfahren bekannt gegeben, nach dessen Ausführung eine sog. ,Keulenschiene“ die Zehe in Dorsalflexion fixiert, um ein Rezidiv zu vermeiden. Die Entstehung der Deformität überhaupt ist nach Klein sch midt auf Kunstfehler in der Behandlung und Verbandtechnik zurückzuführen und wird nach Schwamm sicher vermieden, wenn die Plantarfläche des Gipsverbandes bis zu den Zehenspitzen reicht, während ein Ausschnitt auf der Dorsalseite die Basis der Zehen zur aktiven Dorsalflexion freizulassen hat, eine Verbandtechnik der Wiener Lorenzschen Klinik, bei der niemals eine Beugekontraktur der Zehen entstand.

Die Gipsbehandlung der Skoliosen bereicherte $\mathrm{NuBbaum}$ durch Angabe eines abnchmbaren Gipskorsettes, welches in Suspension angelegt und nach dem Trocknen mit Cramerschienen armiert ist, die halbringförmig un Brust und Taille greifen und vorn mit der Medianlinie mit Öse und Haken geschlossen werden. Obwohl Dauerresultate mit diesem die Bauchatmung einengenden Korsett noch nicht vorliegen, hebt $\mathrm{NuBbaum}$ doch seinen Vorteil hervor, während der Redression Turnübungen zu erlauben, eine lokale Behandlung der Muskeln und der Haut zu ermöglichen, während der Nacht nicht zu belästigen und eine genaue Lokalisation des Druckes zu gestatten. Zur Vorbereitung starrer dreifacher Skoliosen für das Abbotmieder hat Starkes ein Mieder konstruiert, das im wesentlichen aus einem Gipsschultergürtel und einem Gipsbeckengürtel besteht, die beide durch 2 vordere und eine hintere lange Distraktionsklammer nach Prof. Hacken bru ch miteinander verbunden sind. Zwischen den Gipsgürteln liegt eine gut gepolsterte, den vorderen und hinteren Rippenbuckeln angebogene Cramerschiene, die zur Detorsion des Brustsegments mit Bindenzügen gegen die Hackenbruchklammern rotiert wird. Möhring demonstrierte auf dem Orthopädenkongreß 1920 einen Skoliosenstützapparat, der den Vorderteil des Körpers völlig frei läßt und auf den Rippenbuckel mittels Hebelsystems einen starren Pelotendruck ausübt, der besser als ein elastischer Druck vertragen werden soll.

Einen neuen Kopfhaubencerband an Stelle der leicht abrutschenden Mitra gab Härtel an; l. fächerförmig über den Ohren sich kreuzende Zirkeltouren um Stirn und Hinterhaupt, 2. Gänge von der Scheitelhöhe zum Kinn, 3. Zirkel- 
touren wie 1. und Befestigung des Bindenendes, 4. Zurückstreifen der Kinntouren über den Hinterscheitel, ähnlich dem Kinnriemen des Helmes, wodurch der Verband besondere Festigkeit erhalten soll.

Bei einem besonders schweren Fall von Caput obstipum konnte Schanz die Leistungsfähigkeit seines Watteredressionsverbandes zeigen, den er auch zur Behandlung der Spondylitis cervicalis empfiehlt.

Mommsen hat zur Kontrakturbehandlung eine Methode allmählicher unblutiger Redression durch Knebelwirkung und Schraubenzug ausgearbeitet. Bei Hüftbeugekontrakturen z. B. beruht der Erfolg auf einer sehr exakten Beckenfixation und einer exakten Fassung der Gliedabschnitte im Gipsterband, dessen Teile durch dauernde Anwendung einer sehr kleinen Knebelkraft auf sehr beschränktem Wege allmählich gestreckt werden. Die Methode Mom msen wirl von Biesalski warm empfohlen und ist eine wertvolle Bereicherung der Kontrakturbehandlung.

Zur Therapie des schnellenden Fingers legt Heer mann ein Stück Radiergummi auf den Sehnenknoten, drückt es mit einer Klammer nach Art einer kleinen Presse fest und läßt diese Vorrichtung über Tag und Nacht unter leichtem Druck tragen, solange sie keine Empfindlichkeit hervorruft. Nach einigen Tagen soll der Finger frei beweglich sein. In gleicher Weise geht Heermann gegen Narben und Kontrakturen vor. Der Apparat ist zu beziehen durch die Fa. Braun in Melsungen (Hessen-Nassau).

Den Abriß der Fingerstrecksehnen am Endglied, den er sich selbst zugezogen hatte, behandelte Graf 4 mal erfolgreich mit einem aus drei den Phalangen anliegenden, seitlich durch 2 Längsbänder fixierten Metallringen, wobei das Endglied in stärkerer Hyperextension stehen muB zur Näherung der gerissenen Sehnenflïchen. $21 / 2-3$ monatiges Tragen dieses wenig störenden Apparates genügte und brachte ideale Heilung.

6. Belák, Die Wirkungsweise der Verbandstoffe in physiologisch-chemischer Hinsicbt. Biochem. Zeitschr. 96. Heft 4. - 7. Brandes, Behandlung der Osteochondritis deformans jurenilis (Perthes). Dtsch. Zeitschr. f. Chirurg. 15j. 3.-4. Heft. -8 . Brüuke, Ùber die Wirkung komprimierender Verbände anf die Muskulatur nebst einigen Beobachtungen an ischämischen Froschmuskeln. Mitt. a. d. Grenzgeb. d. Mer. u. Chirurg. 31. Jena 1919. - 9. Fränkel, Verbandtechnik bei Luxatio coxae congenita. Zentralbl. f. Chirurg. 1920. Nr. 26a, 690. - 10. Derselbe, Fernresultate bei angeborenem Klumpfuß. Münch. med. Wochenschr. 1920: 525. - 11. Dersel be. Fernresultate beim angeborenen Klumpfub. Zeitschr. f. orthop. Chirurg. 40. 451. - 12. Graf, Ĺber den Abriß der Fingerstrecksehnen am Endglied. Münch. med. Wochenschr. 1920. Nr. 7. 200. - 13. Gretsel, Stanniol als Verbandnittel. Zentralbl. f. Chirurg. 1920. Nr. 26 a. 67\%. - 14. Derselbe, Metallfolien in der praktischen Chirurgie. Dtsch. med. Wochenschr. 1920. Xr. 51. - 15. Härtel, Zur Technik des Kopfhaubenverbandes. Zentralbl. f. Chirurg. 1920. Nr. 47. - 16. Haubenreißer, Das Patellarfenster. ein kleiner Kunstgriff zur Verhütung von Kniegelenksversteifungen infolge Gipsverbandes. Zentralbl. f. Chirurg. 1921. Nr. 8. - 17. Heermann, Zur Therapie dess schnellenden Fingers. Dtsch. med. Wochenschr. 1919. 52. - 18. Kleinschmidt, O., Die typische Beugekontraktur der großen Zehe nach langer Ruhigstellung. Arch. f. orthop. Unfallchirurg. 1S. $120 \mathrm{ff}$. - 19. Derselbe, Die operative Behandlung der typischen Beugekontraktur der großen Zehe. Zentralbl. f. Chirurg. 1920. Nr. 11. 243. - 20. Meyer, Die unblutige Behandlung der angeborenen Hüftrerrenkung. Münch. med. Wochenschr. 1920. 1364. - 21. MIöh ring, Skoliosenstützapparat. Zentralbl. f. Chirurg. 1920. 696. - 22. Mom msen, Methode zur unblutigen allmählichen Redression von doppelseitigen Hiuftbeugekontrakturen. Zentralbl. f. Chirurg. 1920. Nr. 26a. 692. - 23. Derselbe, Das Prinzip der Dauerbelastung in der Kontrakturbehandlung. Zeitschr. f. orthop. Chirurg. 
40. 1 ff. - 24. NuBbaum, Ubber Redression schwerer Skoliosen durch ein abnehmbares Gipskorsett. Zeitachr. f. orthop. Chirurg. 40,258ff. - 25. Peltes ohn, Utber die Erkennung und Behandlung der angeborenen Hüftgelenksverrenkung beim Säugling. Mej. Klinik 1920. Nr. 17. - 26. Propping, Drainage mit Rohgaze. Zentralbl. f. Chirurg. 1920. Nr. 18. 424. - 27. Roederer, Kaseinpulver als Ersatz für Zelluloid der Behelfsapparate. Presse méd. Paris 1920. Nr. 20. - 28. Schanz, Watteverband bei Caput obstipum. Münch. med. Wochenschr. 1920. 1130. - 29. Scheidtmann, Yatrengaze zur Wundbehandlung. Dtsch. med. Wochenschr. 1920. Nr. 26 u. 27. - 30. Schwam m, Eine Bemerkung zum Aufsatz Kleinschmidts: ,C'ber die operative Behandlung der typiscen Bengekontraktur der großen Zehe. Zentralbl. f. Chirurg. 1920. Nr. 26a.676. - 31. Starker, Mieder mit Distraktionsklammern zur Vorbereitung für Abbottmieder. Zentralbl. f. Chirurg. 1920. 833. 32. Veilch en blau, Zur Behandlung des angeborenen Klumpfußes. Dtsch. med. Wochenschr. 1920. Nr. 7.

\section{Verbandtechnisches in der Frakturbehandlung und Extensionsapparate.}

Eine Stellungnahme zur Frakturbehandlung liegt nicht im Rahmen dieses Referates. Dagegen sind eine Reihe von Arbeiten auf diesem Gebiete erschienen, die bei einer Zusammenfassung der orthopädischen Verbandtechnik unbedingt berücksichtigt werden müssen, wenn ein vollständiges Gesamtbild der Fortschritte dieses wichtigen Zweiges der Orthopädie gegeben werden soll.

v. Brunn rühmt und empfiehlt warm die Verwendung ron Gipsschienen, deren Technik überaus einfach und von jedem Arzt leicht zu erlernen ist und eino individuelle Schiene liefert, die nicht nur bei Frakturen, sondern auch bei erforderlicher Fixation von Gliedabschnitten infolge Gelenkentzündungen, nach Operation $u$. dgl. sich bestens bewährt. $\nabla$. Brunn redet einer systematischen Anwendung von Gipsschienenverbänden in der Frakturbehandlung das Wort, zu dem Becker sich zustimmend äu Bert bei Vorderarmbrüchen, während letzterer Autor bei Unterschenkelbrüchen polsterlose Gipsverbände empfiehlt, die nach vorherigem Rasieren der Haut direkt aufgelegt werden und ein Umhergehen gestatten. Einem portativen Verband nach dem Prinzip des bei der Koxitis üblichen Lorenzschen Gehgipsverbandes empfiehlt Chatzkelsohn bei Frakturen der unteren Extremität, wobei er diesen aus einer bis in die Achselhöhle reichenden Außen- und bis zum Tuber ossis ischii reichenden Innenschiene bestehenden Verband mit einer Extension durch Gummizüge kombiniert; Chatzkelsohn war allerdings bisher nicht in der Lage, Röntgenkontrolle auszuüben. In der französischen Literatur spielt zur Zeit der Delbetsche Verband eine Rolle, den Collen in der Presse médicale folgendermaßen beschreibt: Der Verband besteht im wesentlichen aus 4 Gipslonguetten, von denen 2 schmale zu beiden Seiten des Unterschenkels herabgeleitet werden, während 2 breitere zirkulär die beiden Seitenlonguetten am oberen und unteren Umfang des Unterschenkels umfassen, an den sie während des Erhärtens genau auf die unterliegende, nicht gepolsterte Haut anmodelliert werden. Dieser Verband muB unter stärkstem Zug angelegt werden und ermöglicht nach dem Erhärten ein Umhergehen, wobei er Knie- und Sprunggelenke ebenso wie die Sohle völlig frei läßt. Für die untere Extremität beschreibt Faltin 2 Pappschienen, die vor dem Anlegen angefeuchtet, dann rinnenförmig gebogen und mit Binden unter Modellierung an die Extremität fixiert werden, eine hintere Rinnenschiene zur Ruhigstellung des Kniegelenks und eine Fußgelenksschiene, deren Fußteil, 
welcher größer wie gewöhnlich ist, in einem stumpfen Winkel zum Unterschenkelteil steht.

Spelta teilt eine von Taddei angegebene vierseitige Gipsschiene mit, die bei Schulterverletzungen bei horizontal erhobenem Arm angelegt wird, nachdem sie vorher nach den Maßen des Armes auf einem mit Holzpflöcken besteckten Holzbrett vorbereitet ist. Der vertikale Schenkel dieser Schiene lehnt sich an die Crista iliaca an und wird durch Bindentouren nach der gesunden Schulter gehalten, durch Kreistouren an den Rumpf fixiert. Die bei Middeldorfs Triangel mangelnde Fixation von Hand und Vorderarm wird vermieden, während der Oberarm vom Verband freibleiben und massiert werden kann. Einen besonderen Extensionsgipsverband für Humerusfrakturen gibt Abadie an, der in den rechtwinkligen Ellenbogengelenkgipsverband auf der Beugeseite ein Eisenband einläßt, das über die Höhe der Schulter nach oben verläuft, und hier durch Gummizug mit einem in die Achselhöhle sich anstemmenden, gepolsterten Drahtbügel verbunden ist. Utber gute Erfahrungen mit immer gebrauchsfertigen, leichten und spannkräftigen Federextensionsschienen für Finger, Hand und Unterarm berichtet Duncker, während Hans eine alte von Dresmann gelehrte Fingerextensionsmethode ins Gedachtnis zurïckruft, die mittels eines durch das Ende des Fingernagels geführten Seidenfadens, eines kurzen Gummischlauches und einer Fingerbrettschiene am Finger einen kräftigen Zug auszuüben gestattet. Zur Frage Nagel- oder Drahtextension äußert sich Kurt Ansinn, um für die Drahtanwendung wegen der reduzierten Infektions- und Fistelgefahr zu propagieren, während Baumberger eine prägnante Studie zugunsten der Steinmannschen Methode lieferte an Hand eines erschöpfenden Literaturverzeichnisses von 183 Nummern. Zur modernen Extensionsbehandlung, die ja nicht nur bei Frakturen, sondern auch in der Vorbehandlung einzurenkender Hüftluxationen (Fränkel), bei der Streckung von Kontrakturen und in der Nachbehandlung osteoplastischer Operationen eine zunehmende Bedeutung gewinnt, lieferten weitere Beiträge Flenster durch Mitteilung zweijähriger Erfahrungen mit der Schömannschen Doppelzangenextension, deren Technik er genau schildert, Herzberg über Drahtextension am Ort der Wahl, Sommer über eine Scherenschiene mit doppelter Steinmannscher Extension und endlich Riedel und Krogius durch Mitteilung neuer Extensionsschienen.

33. A badie, Appareil à extension continue pour fracture de l'humerus, de fabrication extemporanée. Arch. prov. de chir. 24. Nr. 7. - 34. Ansinn, Kurt, Nagel- oder Drahtextension? Zentralbl. f. Chirurg. 1920. Nr. 11. - 35. Baumberger, Uber Steinmannsehe Nagelextension. Arch. f. orthop. Unfallchirurg. 18. 150. 1920. - 36. Becker, I., Utber die Behandlung von Unterschenkelbrüchen mit polsterlosen Gipsverbänden und über die Behandlung von Unterarmbrüchen mit Gipsschienen. Monatsschr. f. Unfallheilk. u. Invalidenw. 1920. Nr. 6. - 37. v. Brunn, M., Zur Behandlung der Knochenbrüche, insbesondere mit Gipsschienen. Münch. med. Wochenschr. 1920. 2. 42. - 38. Chatzkelsohn, Gehschienen mit elastischer Extension bei Frakturen der unteren Extremität. Dtsch. Zeitsehr. f. Chirurg. 160. 3. -4. Heft. 1920. - 39. Collen, Zur Behandlung der Diaphysenfrakturen der Beine nach Delbet. Presse med. 1920. Paris. - 40. Duncker, tber Federextensionsschienen für Finger-, Hand- und Unterarmbrüche. Münch. med. Wochenschr. 1920. Nr. 28.816. - 41. Faltin, Om pappskenor för de nedre extremiterna. Finska LäLaresellskapets, Handlinger. 62, 396-402. 1920. Nr. 7 u. 8. - 42. Flenster, Frakturenbehandlung mit der Schömannschen Zange. Doppelzangenextension. Berl. klin. Wochenschr. 1920. Nr. 5. - 
Hans Blencke:

43. Hans, Von Nadeln, Füden, Knoten und Nähten. Münch. med. Wochenschr. 1920. 753. - 44. Herzberg, Zur Drahtextension am Ort der Wahl. Zentralbl. f. Chirurg. 1920. Nr. 39. 45. Krogius, Eine Sehiene zur Behandlung von Oberschenkelfrakturen. Finska Läkarescllskapets, Handlinger. 60, 540. - 46. Riedel, A., Eine einfache Extensionsschiene. Inaug.-Diss. Rothenburg 1919. Ref. Zentralbl. f. Chirurg. 1920. 283. - 47. Som mer, R., Behandlung von Unterschenkelbrüchen mit starker Verkürzung. Zentralbl. f. Chirurg. 1920. 504. - 48. Derselbe, Ein Beitrag zur Behandlung stark verkürzter Unterschenkelbrüche nebst Angabe einer neuen Schiene. Bruns Beitr. z. klin. Cliirurg. 118, 126. Heft 1. - 49. Sinlio Spelta, Contributo clinico alla cura delle fratture dell' omero mediante il quadrilatero gosatto. Policlinico, sez. chirurg. 27, 44. 1920.

\section{Portative Apparate.}

Aus Steins Feder ist eine Würdigung des an 16. III. 1918 im Alter von s0 Jahren verstorbenen Hessing erschienen, der mit seinen Schienenhülsenapparaten, seinem Hüftbügelkorsett und seiner Leimverbandtechnik bahnbrechend wirkte und so der Entwicklung der deutschen Orthopädie auf dem Gebiete der Knochen- und Gelenktuberkulose, der ambulanten Frakturbehandlung, der Lähmungen und Kontrakturen und in der Korsettbehandlung durch seine epochemachenden Apparate ein mächtiger Förderer war.

Im ersten Bande seiner Wiederherstellungs- und orthopädischen Chirurgic widmet Deffosses dem Apparatenbau auf Grund der französischen kriegschirurgischen Erfahrungen eingehende Betrachtungen.

ther die Indikationsstellung, ob orthopädische Apparate angewendet werden sollen, äuBert sich Peltesohn bei psychogenen Deformitäten ablehnend wegen der Kosten und wegen der eventuellen Schädigungen in Form von Muskelatrophie und statischen Gelenkerkrankungen, während anderseits Payr und Schanz nach Gelenkmobilisationen an der Tnterextremität vor allem an der Hüfte die Schienenhülsenapparate nicht entbehren können, welche Schan z auch bei Ostitis fibrosa des Beines und bei Schenkelhalsbrüchen mit bestem Erfolge angewandt hat. Bähr gibt allerlei praktische Ratschläge für die Apparatbehandlung der Kontrakturen der verschiedenen Gelenke der Unterextremität und weist auf häufige Fehler in dieser Behandlung hin.

Bei der habituellen Schulterverrenkung hat $B 0 \mathrm{hm}$ einen einfachen Apparat angegeben, der so konstruiert ist, daß man durch geeignete Anschläge isoliert die Vorwärts- oder Rückwärtshebung oder die Abduktion des Armes in jedem gewünschten Winkel zum Stehen bringen kann, während Wideroë zur funktionellen Behandlung der Klavikularbrüche eine Bandage hat machen lassen, die aus Leibgürtel, steifer Rückenstütze, 2 Schulterblattpelotten und Schulterriemen bestehend eine feste Fixation der Fraktur gestatten und sofortige schmerzlose Arbeitsfähigkeit ermöglichen soll. Zur Behandlung des Ellenbogenschlottergelenks demonstrierte Goetze einen Schienenhülsenapparat, der in einem Hautlianal zwischen der Bizeps- und Trizepsgruppe Halt gewinnt und mit gutem Resultat getragen werden soll.

Zum Kapitel der Lähmungsprothesen hat v. Recklinghausen ein zweibändiges Werk herausgebracht, das für dieses Gebiet und die Gliedermechanik als klassisch bezeichnet werden nuß. Die Hauptaufgabe der Lähmungsprothese erblickt v. Recklinghausen darin, dem gelähmten Glied wieder zu möglichst weitgehender Gebrauchsfähigkeit zu verhelfen, dem 
Patienten zu tunlichster Arbeitstüchtigkeit, durch Anbringung künstlicher Muskeln in Form von Stahlfedern. Das Irleal sei nicht ein, sondern eine ganze Reihe, ein ganzes System bester Apparate für ein und dieselbe Lähmung. Eine Ubersicht über die gebräuchlichsten Radialis-, Peroneus- und Ulnarisschienen gab Geinitz in seiner Abhandlung über Nervenschüsse, während Paramelle verschiedene französische Apparate bei Peroneus-, Tibialislähmung usw. beschreibt, die alle das Gemeinsame haben, daß vermittels eines Schultergürtels als Aufhängepunkt die Schulter gewählt wurde. Roederer und Demoulin bearbeiteten 3 verschiedene Prothesen für Ulnaris-Mredianuslähmungen, von denen sie einen mit Riemen versehenen deutschen Arbeitshandschuh für landwirtschaftliche Arbeiter, ,trotz seines Ursprunges" wegen seiner großen ZweckmäBigkeit ihren französischen Kollegen empfehlen. Von Bidon stammen sehr verwickelte, für das tägliche Leben zu empfindliche Konstruktionen von Linterstützungsgeräten Gelïhmter, die Jalaguier beschreibt, ein Autor, der auch eine Peroneusschiene veröffentlicht hat, welcher die Vorzüge der Leichtigkeit, Haltbarkeit und Unsichtbarkeit zugeschrieben werden. Eine Aufzählung von 21 deutschen Rarlialisschienen verschiedener Herkunft findet sich im Jahresbericht von Radike über die Tätigkeit der Prüfstelle für Ersatzglieder zu Berlin. Auf dem Orthopädenkongre 31920 zu Dresden demonstrierte Göcke eine anscheinend sehr brauchbare, durch Schulterhebung betätigte Bandage zur Unterschenkelstreckung bei Quadrizepslähmung.

Die Plattfulbehandlung mittels Sehuhwerks durch Erhöhung des Absatzes, nicht auch des Vorderschuhes, auf der Innenseite um $1 \mathrm{~cm}$ beschreibt Heermann, ohne damit etwas Neues zu bringen. Einen regelrecht heilenden Einfluß schreibt Romich seiner verschieblich federnden Einlage zu, welche sich beim Gehen und Stehen der wechselnden Verlagerung der Belastung des Fußgewölbes und damit seiner weehseInden Form dauernd anpassen soll, muß jedoch Fischers Entgegnung erfahren, daß dieses pathologische Spiel der Fußgelenke die weitere Iockerung des Bandapparates verursache. Dem $Z$ weifel $F$ ischers, oh es möglich sei, die Plattfußfrage durch fabrikmäßig hergestellte, fertig verkäufliche Einlagen zu lösen, kam man nur zustimmen. Zur Messung und Behandlung des Knickfußes licferte Iselin einen mit zahlreichen Abbildungen ausgestatteten Beitrag, dem er eine Besprechung und Kritik des Leistens und des Stiefelbaues des eidgenössischen Wilitär'schuhes anschloß.

Anscheinend sehr praktische, portative Ubungsapparate für Amputierte mit Muskelkanälen nach $S$ auerbruch mit einer die Arbeit kontrollierenden Meßbüchse beschreibt Blumenthal.

$\mathrm{Br}$ üu ing veröffentlichte einen neuen optischen Meßapparat zum Zeichnen von Skoliosen, X-, O-Beinen und anderen Deformitäten, der sich auch bei der Begutachtung Unfallverletater gut bewährt hat und eine sichere Kontrolle über Behandlungsresultate ermöglichen soll.

Von Mollenhauer stanmt die Angabe einer neuen Bruchbandage, die er gemeinsam mit der Firma H. Loewi-Berlin konstruiert hat und die sich besonders bei großen Skrotalhemien bewährt haben soll. Die neue Pelotte reitet sozusagen auf dem Schambeinast und wird von ihm derart suspendiert, daß eine Versehiebung unmöglich sein soll. 
50. Bähr, Die Versorgung der Beinkontrakturen mit Prothesen. Dtsch. med. Wochenschrift 1920. 6. - 51. Bid ou, De l'orthopédie instrumentale. Bull. l'acad. de méd. Paris. 83. Jahrg. 81, 286. Nr. 10. - 52. Blumenthal, Tragbare Cibungsapparate für Amputierte mit Muskelkanälen nach Sauerbruch. Berl. klin. Wochenschr. 1919. 45. - 53. Böh m, M., Die mechanische Behandlung der habituellen Schulterverrenkung. Berl. klin. Wochenschr. 1920. 15. - 54. Brüning, Ein neuer optiseher Meßapparat. Zentralbl. f. Chirurg. 1920. Nr. 31. 948. - 55. Deffosses, Chirurgie réparatrice et orthopédique. Paris. Masson \& Cie. 1920. - 56. Fischer, Ernst, Zur Plattfußeinlage. Dtsch. med. Wochensehr. 1920. Nr. 28. - 57. Geinitz, Die Nervenschüsse. Ergebn. d. Chirurg. u. Orthop. 12. Berlin 1920. - 58. Göcke. Willkürlich bewegliche Bandage bei Quadrizepslähmung. Zentralbl. f. Chirurg. 1920. 695. - 59. Goetze, Schienenhïlsenapparat bei Ellenbogenschlottergelenk. Münch. med. Wochenschr. 1920. 855. - 60. Heermann, Zur Behandlung des Plattfußes. Dtsch. med. Wochenschr. 1920. 6. - 61. Jalaguier, Sur un ouvrage du Dr. Gabriel Bidou, concernaut l'orthopédie instrumentale. Bull. l'academie de méd. Paris 81. Nr. 24. 197. 62. Derselbe, Sur un appareil de prothèse de Poujoula et Menropian pour la paralysie du nerf sciatique poplité externe. Bull. de l'acad. de méd. Paris. 82. Jahrg. 80. 252. 63. Iselin, Zur Messung und Behandlung des Knickfußes. Schweiz. med. Wochenschr. 1920. Nr. 5. Ref. Zentralbl. f. Chirurg. 1920. 1031. - 64. Mollenhauei', Fine neue Bruchbandage mit Suspension am horizontalen Schambeinast. Münch. med. Wochenschr. 1921. Nr. 8. - 65. Paramelle, Appareillage des quelques lésions musculaires ou nerveuses à l'aide d'appareils à sangles. Revue d'orthop. o7. 1920. Heft 1. - 66. Payr, Zehn Jahre Arthroplastik. Zentralbl. f. Chirurg. 1920. Nr. 14. - 67. Peltes ohn, Utber die Verkennung der Psychogenie von Deformitäten. Berl. klin. Wochenschr. 1919. 36. - 68. Radike, Mitgliederversammlung der Prüfstelle f. Ersatzglieder zu Berlin (Radialisschienen). Arch. f. orthop. Unfallchirurg. 1920. 257. - 69. v. Recklinghausen, Gliedermechanik und Lähmungsprothesen. Berlin 1920. Julius Springer. - 70. Roederer et Dumoulin, Trois appareils de prothèse fonctionelle pour paralysies médio-cubitale. Presse méd. Paris. 89. 1920. - 71. Romich, Eine neue Plattfußeinlage. Dtsch. med. Wochenschr. 1920. Nr. 14 u. 15. - 72. Schanz, Demonstrationen. Nünch. med. Wochenschr. 1920. 1130. - 73. Stein, Friedrich v. Hessing und die deutsche Orthopädie. Zeitschr. f. orthop. Chirurg. 39. 1. Heft. - 74. Widerö̈, Zur funktionellen Behandlung der Klavikulabrüche. Zentralbl. f. Chirurg. 1920. Nr. 23.

\section{Medikomechanische Apparate.}

Am Eingang dieses Kapitels sei der bedauerlichen Tatsache Erwähnung getan, daß infolge der schlechten Zeitverhältnisse das Zentralblatt für chirurgische und mechanische Orthopädie sein Erscheinen hat einstellen müssen.

Desgleichen sei an dieser Stelle ein von v. Bezold und Walb unterzeichneter Aufruf erwähnt, die Abteilung für medikomechanische Apparate im Germanischen Museum in Nümberg durch Zuweisung gecigneter Stücke zu vervollständigen.

Den Fragen der Mechanotherapie widmet sich die ärztliche Gesellschaft für Mechanotherapie, auf deren Jahresversammlung $1920 \mathrm{Hirsch}$ die Mechanotherapie im Rahmen der Kurortbehandlung und Blumenthal die Mechanotherapie der Amputierten behandelte. Guradze nahm zur Frage Chronische Rheumatismen und Mechanotherapie Stellung, ein Gebiet, welches Smitt vom Standpunkt der Mechanotherapeuten an anderer Stelle an diagnostischen Irrtümern bei Muskelrheumatismus beleuchtete. Das Verhalten der Körpertemperatur bei mechanotherapeutischer Behandlung untersuchte der Skandinavier Rancken, der bei Rektalmessungen eine Erhöhung um höchstens $0,07^{\circ} \mathrm{C}$ bei mechanotherapeutischer Behandlung gegen eine solche $0,1-0,7^{\circ} \mathrm{C}$ bei gymnastischen Übungen feststellte. 
Finen neuen Fingerpendelapparat gab Schütz an, während der Schweizer v. Mandach einen MeB- und Utbungsapparat für Pro- und Supinatión der Hand konstruierte und Lavermicocca auf dem Kongreß der italienischen orthopüdischen Gesellschaft Oktober 1919 einen leicht zu bauenden Apparat vorschlug, der bei posttraumatischen Deformitäten der Finger zur Anwendung zu kommen hätte. Lavermicocca legte außerdem einen Apparat mit elastischen Zïgen vor, der es ermöglicht, den FuB aktive und passive Bewegungen in allen seinen Gelenken bei guter Fixierung des Unterschenkels ausführen zu lassen. Neergard beschreibt einige medikomechanische Apparate, die von jedem Handwerker leicht hergestellt werden können und aus Kombinationspendel für Ellbogen-, Knie- und Sprunggelenk, Kombinationspendel für Handgelenk-Adduktion und -Abduktion bzw. -Beugung, -Streckung und Fußpendel, Apparat für Pro- und Supination und Glühlicht.Heißluftkasten bestehen; ihre Konstruktion muß im Original nachgelesen werden.

Eine ausführliche Arbeit über die medikomechanische Behandlung der Frakturen im Krankenhause Bergmannsheil zu Bochum lieferte Seele und beweist damit die hohe Bedeutung der aktiven und passiven Bewegungen für die funktionelle Frakturbehandlung, die jetzt an Stelle der bisher üblichen fixierenden Gipsverbände getreten ist.

75. v. Bezold und Walb, Sammlung ärztlicher Apparate im germanischen Museum in Nürmberg. Münch. med. Wochenschr. 1920. 710. - 76. Blumenthal, Die Mechanotherapie der Amputierten. Münch. med. Wochenschr. 1920. 170. - 77. Gurad ze, Chronische Rheumatismen und Mechanotherapie. Münch. med. Wochenschr. 1920. 170. - 78. Hirsch, 2. Jahresversammlung der ärztlichen Gesellschaft für Mechanotherapie am 10./11. I. 1920, ebenda. - 79. Dersel be, Mechanotherapie im Rahmen der Kurortbehandlung. Münch. med. Wochenschr. 1920. 170. - 80. Lavermicocca, Apparat für die frühzeitige Nachbehandlung bei den funktionellen Läsionen des Fußes. Zeitscbr. f. orthop. Chirurg. 40.500. - 81. Dersel be, Funktionelle Wiederherstellung der Hand durch ein einfaches mechanisches Hilfsmittel; ebenda. - 82. v. Mandach, Ein MeB- und Utbungsapparat für Pro- und Supination der Hand. Schweiz. med. Wochenschr. 1920. Nr. 17. - 83. Neergard, Einige medikomechanische Improvisationen mit Angabe eines einfachen Licht-HeiBluftkastens. Schweiz. med. Wochensehr. 1920. Nr. 4. - 84. Rancken, Das Verhalten der Körpertemperatur bei mechanotherapeutischer Behandlung und gymnastischen Freiübungen. Skandinav. Arch. f. Physiol. 1920. 40. - 8j. Sc h üt z. Fingerpendelapparat. Münch. med. Wochenschr. 1920. 170. - 86. Seele, Uber die medikonechanische Behandlung der Frakturen in Krankenhause Bergmannsheil zu Bochum. Monatsschr. f. Lnfallheilk. u. Invalidenw. 1920. Nr. 6. - 87. Smitt, Mechanotherapie und diagnostische Irrtümer bei Muskelrheumatismus. Münch. med. Wochensehr. 1920. 606. 